\title{
Urban Transitions in Pandemic Scenarios: Insights from Hong Kong
}

\author{
Mirna Zordan, Jin Yeu Tsou \\ Department of Architecture and Civil Engineering, \\ City University of Hong Kong, Hong Kong \\ mzordan2-c@my.cityu.edu.hk; jytsou@cityu.edu.hk \\ Tel: +85260985625
}

\begin{abstract}
Pandemic scenarios are affecting the psychological stability of the worldwide urban population, consequently impacting, among others, on human behaviour towards the use of urban spaces. This study explores linkages between psychological dynamics and uses of urban spaces in Hong Kong under the COVID-19-due psychological distress. Significant transitions in urban spaces preferences and decision-making processes emerged in favour of outdoor-related environmental factors, the noncoexistence of necessary and social activities in the same area, and the prevalence of behavioural imitators rather than initiators. A significant result revealed the relevance in urban dwellers perceptions' for activities rather than places.
\end{abstract}

Keywords: urban transitions; mass psychology; Covid-19; Hong Kong;

eISSN: 2398-4287C 2020. The Authors. Published for AMER ABRA cE-Bsby e-International Publishing House, Ltd., UK. This is an open-access article under the CC BYNC-ND license (http://creativecommons.org/licenses/by-ncnd/4.0/). Peer-review under responsibility of AMER (Association of Malaysian Environment-Behaviour Researchers), ABRA (Association of Behavioural Researchers on Asians) and cE-Bs (Centre for Environment-Behaviour Studies), Faculty of Architecture, Planning \& Surveying, Universiti Teknologi MARA, Malaysia.

DOI: https://doi.org/10.21834/jabs.v5i17.376 


\subsection{Introduction}

Psychological needs and perceptionsare the major driving forces lingering behind the extremely complex mechanism of human behaviour. Behavioural dynamics in relation to the physical environment have been widely investigated over decades, trying to extrapolate basic principlesaffecting both space and human attitudes. The results achieved nowadays are remarkable. However, in the perspective of this field of investigation, less attention has been dedicated to psychological needs and perceptions affecting human behaviour 'a priori', and most importantly all the theories explored are based on behavioural attitudes driven by standard or conventional psychological conditions. Hence, the relationship between the physical environment and human behaviour can be dramatically questioned and re-discussed if an unexpected distortion of mass psychological perception occurs. The spread of a pandemic can induce this distortion. During a pandemic outbreak, the immediate researchers'responsesare usually focusing on the investigation of the physical spread of the disease and its contagion dynamics and modelling, thus concerning majorly epidemiological and medical studies. However, another typology of contagion rather than physical is spreading whenever a pandemic occurs. The spread of the disease in some cases impacts the most psychological conditions and perceptions of the affected population rather than the effective dissemination of the disease itself (Dodds \& Watts, 2004; Epstein et al., 2008; Huremovic, 2019), endorsing and questioning the power of behavioural contagion in impacting on human attitudes, and consequently on urban dynamics and public life. However, no attention has been given to the effects that a pandemic, and especially behavioural contagion, can arise in high-density environments towards the use of the public realm within the cities' space as yet.

The role of public spaces in easing difficulties and issues related to everyday life is worldwide recognised. Their impact on wellbeing and quality of life is demonstrated as affecting mental, physical, and social conditions of urban dwellers by an enormous quantity of studies (Bishop \& Marshall, 2017; Francis, Giles-Corti, Wood, \& Knuiman, 2012; Hanan, 2017; Nasution \& Zahrah, 2018; UN-Habitat, 2013; Villanueva et al., 2015, among others). This relationship with health-related outcomes andthe complexity and multidimensionality of public spaces advocate them as essential elements within city spaces, especially where scarcity of land and available space is a contemporary issue. The provision and management of public spaces are increasingly becoming problematic and difficult to operationalise (Carmona, 2010), especially in high-density environments. To maximise the efficiency of public space distribution and minimise the impact on available land,

it is fundamental to investigate behavioural patterns towards different public spaces typologies and to understand the urban population perceptions and preferences among them to develop better redistribution and development strategies (Zordan, 2018). If these patterns have been widely explored under common or standard psychological conditions, there is no study that considers extreme situations, where mass psychological distress is acting as a driving force affecting behavioural patterns towards the public realm.

The contribution of this researchconsists of starting the exploration of mass psychological distressimpact on different uses of urban spaces in Hong Kong, and decision-making 
processes behind them. The investigation of preferences and decision-making dynamics before and after the spread of Covid-19 will allow initial detection of transitional processes and behavioural dynamics in Hong Kong urban population, emphasising the current need of attention of mass psychological distress in urban environmental studies. This study has two objectives. Firstly, it will detect transitions in preferences and activities among different typologies of urban spaces before and after the spread of Covid-19 trying to extrapolate initial tendencies on which spaces are more resilient than others. This, to get an initial portrait of behavioural dynamics leading urban dwellers to choose some urban spaces rather than others. Secondly, the study will focus on the decision-making processes lying behind those preferences by investigating psychological attitudes deriving from behavioural contagion theory principles. These psychological and behavioural dynamics towards the urban environment will point out population needs, allowing primordial evaluation tools about current urban spaces (concerning population needs fulfilment), and suggesting directions for the design of future urban spaces. The main questions this study is aiming at exploring are

(1) Which urban spaces, behavioural patterns, and activities are 'surviving' the transition?

(2) Which are the urban spaces preferences of the urban population before and after the spread of the COVID-19?

(3) What is the role of behavioural contagion dynamics during the decision-making process?

(4) What are the implications for the usage of the public realm in Hong Kong?

\subsection{Literature Review}

The complex system holding together the relationships between human behaviour and physical environment with the influence of psychological dynamics is synthesised in Fig.1. Psychological factors derive from a previous emotional state that consciously or subconsciously is present in the individual. Whenever looking at external factors inducing emotional states, two types of conditions emerge, conventional (everyday life) or nonconventional (distress) conditions. As psychological dynamics are at the basis of every action, and in the case of extreme distress affecting an entire population, if not the world as a whole, those mass psychological factors decisively determine consequences on human behaviours and attitudes.In the case of a pandemic, contagion occurs not only related to the physical transmission of the disease. Psychological perceptions, such as fear and perceived risks, in some cases are spreading faster than the disease itself (Epstein et al., 2008; Rubin et al., 2009). Thus, the consequences for human perceptions are undeniably impacting on behavioural dynamics. In this scenario, the word 'contagion' appears to be the most appropriate to define dynamics related to both physical and psychological transmission of states. Contagion theory refers to collective behaviours that investigates how the crowd, or mass, can affect individuals behaviours (Huremovic, 2019; Le Bon, 1896), and its psychology can be primarily divided into emotional and behavioural contagion (Hatfield et al., 1992; Huremovic, 2019; Wheeler, 1966). Hence, when investigating human behaviour in relation to the built environment, the phenomenon of behavioural contagion draws attention into the field. Defined firstly in 1950 as an event in which a recipient's behaviour has changed to become 
"more like" that of the actor or initiator. This change has occurred in a social interaction in which the actor has not communicated intent to evoke such a change(Polansky, Lippitt, \& Redl, 1950, p.322), behavioural contagion definition did not distinguish from other types of social influence. To keep the definition still open, Wheeler (1966), applied a reduction of sentences (Carnap, 1936, 1937; Wheeler, 1966)to keep using the word 'behavioural contagion'. The Encyclopaedia of Social Psychology defines it more synthetically as the tendency of people to repeat behaviour after others have performed it (van Baaren, 2007). For behavioural contagion to occur, there is a need of face to face interaction; it usually occurs subconsciously, and it requires at least one imitator and one initiator. It has been studied that density and numbers are two factors affecting the spread of behaviour and facilitating the transmission (Freedman et al., 1980). The principles on which behavioural contagion is based are four(Redl, 1949; Wheeler, 1966):

1) acute conflict in the area within the imitator;

2) a high-degree liability of personal balance;

3) strong urge towards impulse expression along the same line of initiator;

4) impulse satisfaction of initiator without fear of guilt.

Despite the enormous amount of studies that applied the concept of behavioural contagion in different fields were mostly related to medical sciences (Suzuki et al., 2016), sociology and psychology (Shirado et al., 2020), and transportation research (Allen et al., 2019), to mention few, however, there is no application of this theory in urban studies specifically looking at the influence of behavioural contagion on decision-making processes related to the use of urban spaces under mass psychological distress (Bish \& Michie, 2010; Jones \& Salathé, 2009; Rubin et al., 2009). The understanding of decision-making processes regarding preferences related to the public realm can give precious hints on the urban design guidelines related to public spaces characteristics.

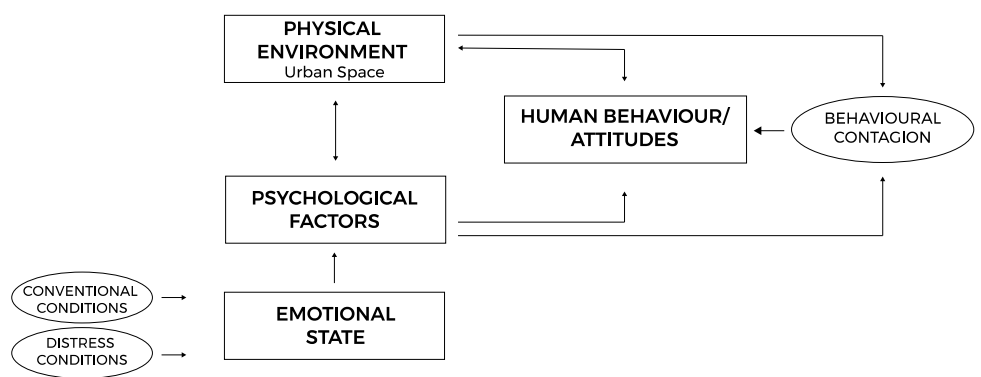

Fig. 1: Conceptual framework of the study. (source: Mirna Zordan)

\subsection{Methodology}


The project here presented follows principles related to exploratory research and methodology, and it concerns a pilot study conducted between the $6^{\text {th }}$ and the $19^{\text {th }}$ of April 2020 right after the spread of Covid-19 in Hong Kong. The study is divided into two main stages following the research objectives; the first with the purpose of detecting behavioural patterns transitions, the second focusing on the investigation of the decision-making processes behind them. The categorisation of public spaces was made based on accessibility and intersubjectivity criteria, excluding the intertwine between public and private (Carmona, 2010; Kohn, 2004); management, ownership, and land value are not considered in this preliminary stage. Due to the nature of the research, survey research design and in-depth interviews were selected as principal methods of investigation. The target of the pilot study analysed in this paper was set to Hong Kong young generations, specifically university students, due to data availability and sample convenience given by the current Covid-19 situation that did not allow on-site research-related activities.

\subsection{Data collection}

Due to exploratory research principles, this study does not aim at inferential analysis; therefore, non-probability sampling was adopted (Saunders, Lewis, \& Thornhill, 2009), by applying a convenience self-selection sampling technique (Saunders et al., 2009). The study's need for cases was advertised among university students by emails and invitations from colleagues and friends; data were collected only from respondents (Saunders et al., 2009).

\subsection{Data analysis}

Data analysis was carried out in two stages. The first was dedicated to the survey data and the detection of patterns with a preference analysis. The second part mostly involved in-depth interviews focused on decision-making processes and questions related to behavioural contagion dynamics. The survey was designed into two sections, one before the spread of the COVID-19 and one at the present time. Questions were related to the use of the public realm in Hong Kong and preferences/frequencies of urban spaces typologies for stationary (social/optional) activities (Gehl, 1987; Ghel \& Svarre, 2013; Mehta, 2006, 2008; Mehta \& Bosson, 2018; W. H. White, 1980). The measurement considered more appropriate was set on Likert-type items (Boone \& Boone, 2012)in most questions. Based on this decision, data analysis was based on the school of thoughts that treats Likert-type items as ordinal variables (Boone \& Boone, 2012), significance was tested with the Chi-square test. Following the survey, in-depth interviews were conducted based on an intentional bias after compiling the survey. The principles related to behavioural contagion theory were preliminarily tested by asking specific questions related to choices and psychological dynamics. Important parameters to be detected were hesitation in decision-making and decisiveness in selfinitiative behaviour.

\subsection{Results}


Demographics data of survey respondents $(n=113)$ revealed an approximately equal gender distribution as $44 \%$ were maleand $56 \%$ were female university students, aged mostly between $15-24(57 \%)$, and $25-34(41 \%)$, with a minor percentage, ranged in $35-44$ years old (3\%). As the target was set on university students, level of education only comprehends junior college or undergraduate $(46 \%)$, and postgraduate and above $(54 \%)$. The majority of respondent were from Hong Kong (48\%), and Mainland China (33\%), with smaller percentages from South East Asia (6\%), Africa (6\%), Europe (3\%), Central Asia (1\%), US (1\%), and Russia $(1 \%)$.

\subsection{Activities, urban spaces and frequencies}

A visual portrait of activities and frequencies are immediately highlighting the dropping of high and medium frequencies of social and optional activities, and the enhancement of low frequencies spreading around both necessary and social or optional activities, see Fig.2 and Fig.3. Percentages are shown in Table 1. The highest decrease of frequency reached the null value in hanging out with friends $(n=112$, from $7 \%$ to $0 \%$ ), and sport ( $n$ (before) $=111,6.31 \%$, to $n=112$ (after), $0 \%$ ). Values between $0 \%$ and $1 \%$ before the spread of Covid- 19 are only related to never going out, never going out for lunch or dinner $(n=112,0 \%)$ and never going out for buying food $(n=112,0.89 \%)$. After the spread, the same interval values are related to always going out ( $n=113,0.88 \%)$, always going out for shopping ( $n=112,0.89 \%)$, never and often going out for public services ( $n=112,0.89 \%$ and $0.89 \%$ ), never going out with friends $(n=112,0 \%)$ and family $(n=112,0.89 \%)$, never going out for sports $(n=112,0 \%)$, and never going out without plans $(n=112,0.89 \%)$. The only activities that reached $0 \%$ are sport and hanging out with friends. The highest percentages reached (over $40 \%$ ) are related mostly to low frequencies (rarely and never). Before the spread of Covid-19, low frequencies are mostly concerning the use of public services ( $n=110,44.5 \%)$, and going out without plans $(n=11$, $45.5 \%)$, while after the spread of Covid-19 percentages relate to going out ( $n=113,75.22 \%)$, going out for lunch or dinner ( $n=112,43.75 \%)$, going out for shopping ( $n=112,46.43 \%)$, use of public services ( $n=112,45.54 \%$ for rarely, $40.18 \%$ for never), hanging out with family $(n=112,62.50 \%)$, sport $(n=112,46.43 \%)$, getting fresh air $(n=112,40.18 \%)$, and going out with no plans $(n=112,50 \%)$.

Frequencies for public spaces usage revealed the same tendency in decreasing high frequencies and increasing low frequencies, as shown in Fig.3 and Fig.4. The more drastic change in frequencies is shown in the shopping mall category regarding the medium-low rate (rarely). Interestingly and against expectations, the frequencies related to urban green spaces did not increase after the spread of COVID-19, following the overall tendency that portraits an incremental decrease of high and medium-high rates among all the typologies of public space. However, a minimum change in trend is revealed regarding playgrounds, switching from a $0 \%$ before the spread to a small increment after the spread in regard to high frequency (always), but still maintaining the decrement in the other rates. Regardless, the highfrequency change is too minimal to be considered as relevant. 
Zordan, M., \& Yeu Tsou, J. / Journal of ASIAN Behavioural Studies (JABs), 5(17) Sep / Dec 2020 (pp. 55-70)

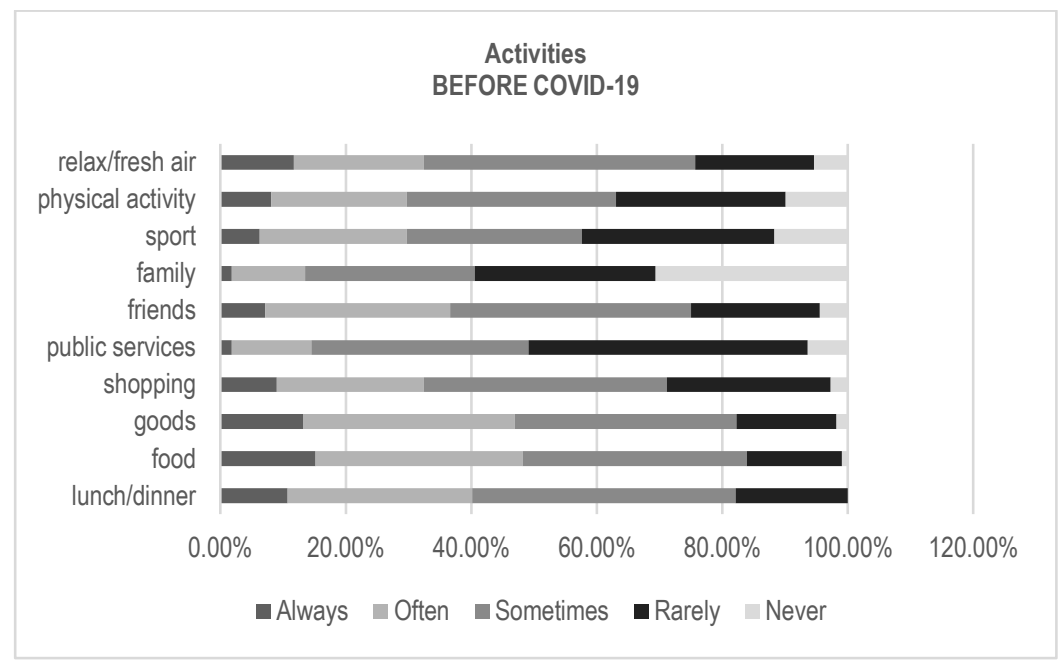

Fig. 2: Why did you go out (except for work and school related activities)? (source: Mirna Zordan)

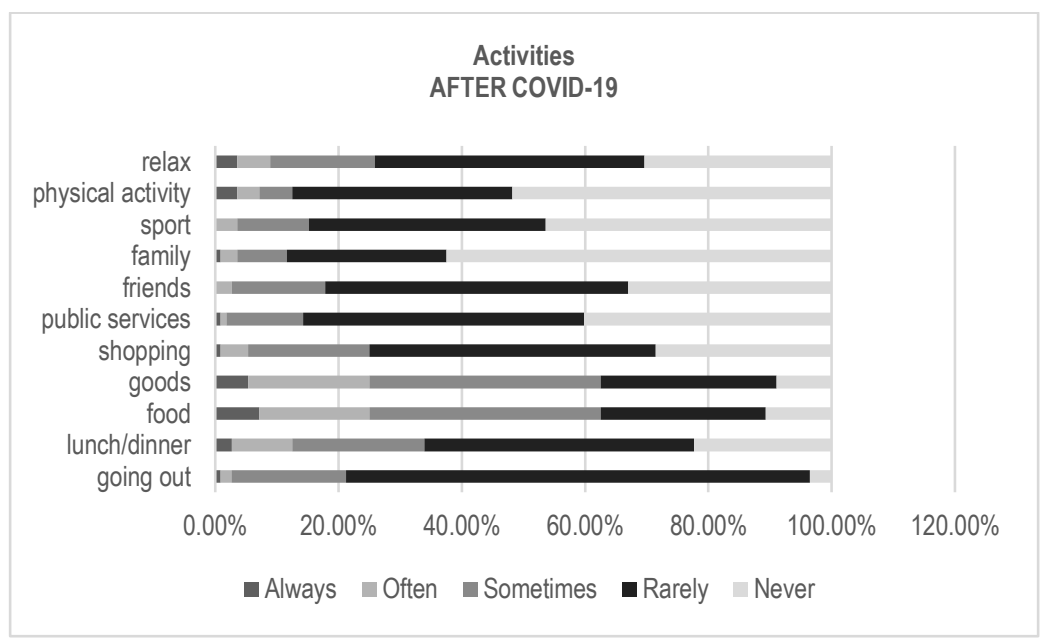

Fig.3: Why do you go out now (except for work and school related activities)? (source: Mirna Zordan) 
Table 1. Percentages, median( $m)$, and mode $(m)$ of frequencies and activities before(b) and after(a) Covid-19 outbreak.

\begin{tabular}{|c|c|c|c|c|c|c|c|c|}
\hline Question & $\mathrm{b} / \mathrm{a}$ & (n) & $\mathrm{m} / \mathrm{m}$ & Always & Often & Sometimes & Rarely & Never \\
\hline \multirow[t]{2}{*}{$\begin{array}{l}\text { How often do you } \\
\text { go out }\end{array}$} & $b$ & 113 & $4-4$ & $7,08 \%$ & $48,67 \%$ & $38,05 \%$ & $6,19 \%$ & $0,00 \%$ \\
\hline & a & 113 & $2-2$ & $0,88 \%$ & $1,77 \%$ & $18,58 \%$ & $75,22 \%$ & $3,54 \%$ \\
\hline \multirow[t]{2}{*}{ Lunch/dinner } & $b$ & 112 & $3-3$ & $10,71 \%$ & $29,46 \%$ & $41,96 \%$ & $17,86 \%$ & $0,00 \%$ \\
\hline & a & 112 & $3-3$ & $2,68 \%$ & $9,82 \%$ & $21,43 \%$ & $43,75 \%$ & $22,32 \%$ \\
\hline \multirow[t]{2}{*}{ Buying food } & $b$ & 112 & $3-3$ & $15,18 \%$ & $33,04 \%$ & $35,71 \%$ & $15,18 \%$ & $0,89 \%$ \\
\hline & a & 112 & $3-3$ & $7,14 \%$ & $17,86 \%$ & $37,50 \%$ & $26,79 \%$ & $10,71 \%$ \\
\hline \multirow[t]{2}{*}{ Buying goods } & $b$ & 113 & $3-3$ & $13,27 \%$ & $33,63 \%$ & $35,40 \%$ & $15,93 \%$ & $1,77 \%$ \\
\hline & a & 112 & $2-2$ & $5,36 \%$ & $19,64 \%$ & $37,50 \%$ & $28,57 \%$ & $8,93 \%$ \\
\hline \multirow[t]{2}{*}{ Public services } & $b$ & 110 & $3-3$ & $1,82 \%$ & $12,73 \%$ & $34,55 \%$ & $44,55 \%$ & $6,36 \%$ \\
\hline & a & 112 & $2-2$ & $0,89 \%$ & $0,89 \%$ & $12,50 \%$ & $45,54 \%$ & $40,18 \%$ \\
\hline \multirow[t]{2}{*}{ Shopping } & $b$ & 111 & $2-2$ & $9,01 \%$ & $23,42 \%$ & $38,74 \%$ & $26,13 \%$ & $2,70 \%$ \\
\hline & a & 112 & $2-2$ & $0,89 \%$ & $4,46 \%$ & $19,64 \%$ & $46,43 \%$ & $28,57 \%$ \\
\hline \multirow[t]{2}{*}{$\begin{array}{l}\text { Hanging out with } \\
\text { friends }\end{array}$} & b & 112 & $3-3$ & $7,14 \%$ & $29,46 \%$ & $38,39 \%$ & $20,54 \%$ & $4,46 \%$ \\
\hline & a & 112 & $1-1$ & $0,00 \%$ & $2,68 \%$ & $15,18 \%$ & $49,11 \%$ & $33,04 \%$ \\
\hline \multirow[t]{2}{*}{$\begin{array}{l}\text { Hanging out with } \\
\text { family }\end{array}$} & $b$ & 111 & $2-1$ & $1,80 \%$ & $11,71 \%$ & $27,03 \%$ & $28,83 \%$ & $30,63 \%$ \\
\hline & a & 112 & $2-1$ & $0,89 \%$ & $2,68 \%$ & $8,04 \%$ & $25,89 \%$ & $62,50 \%$ \\
\hline \multirow[t]{2}{*}{ Sport } & $b$ & 111 & $3-2$ & $6,31 \%$ & $23,42 \%$ & $27,93 \%$ & $30,63 \%$ & $11,71 \%$ \\
\hline & a & 112 & $1-1$ & $0,00 \%$ & $3,57 \%$ & $11,61 \%$ & $38,39 \%$ & $46,43 \%$ \\
\hline \multirow[t]{2}{*}{ Physical activity } & $b$ & 111 & $3-3$ & $8,11 \%$ & $21,62 \%$ & $33,33 \%$ & $27,03 \%$ & $9,91 \%$ \\
\hline & a & 112 & $2-2$ & $3,57 \%$ & $3,57 \%$ & $5,36 \%$ & $35,71 \%$ & $51,79 \%$ \\
\hline Relax/fresh air & $b$ & 111 & $3-3$ & $11,71 \%$ & $20,72 \%$ & $43,24 \%$ & $18,92 \%$ & $5,41 \%$ \\
\hline Relax & a & 112 & $2-2$ & $3,57 \%$ & $5,36 \%$ & $16,96 \%$ & $43,75 \%$ & $30,36 \%$ \\
\hline Fresh air & a & 112 & $1.5-1$ & $2,68 \%$ & $10,71 \%$ & $19,64 \%$ & $40,18 \%$ & $26,79 \%$ \\
\hline \multirow[t]{2}{*}{ No plans } & $b$ & 111 & $2-2$ & $2,70 \%$ & $17,12 \%$ & $27,93 \%$ & $45,05 \%$ & $7,21 \%$ \\
\hline & a & 112 & $2-2$ & $0,89 \%$ & $3,57 \%$ & $15,18 \%$ & $30,36 \%$ & $50,00 \%$ \\
\hline
\end{tabular}




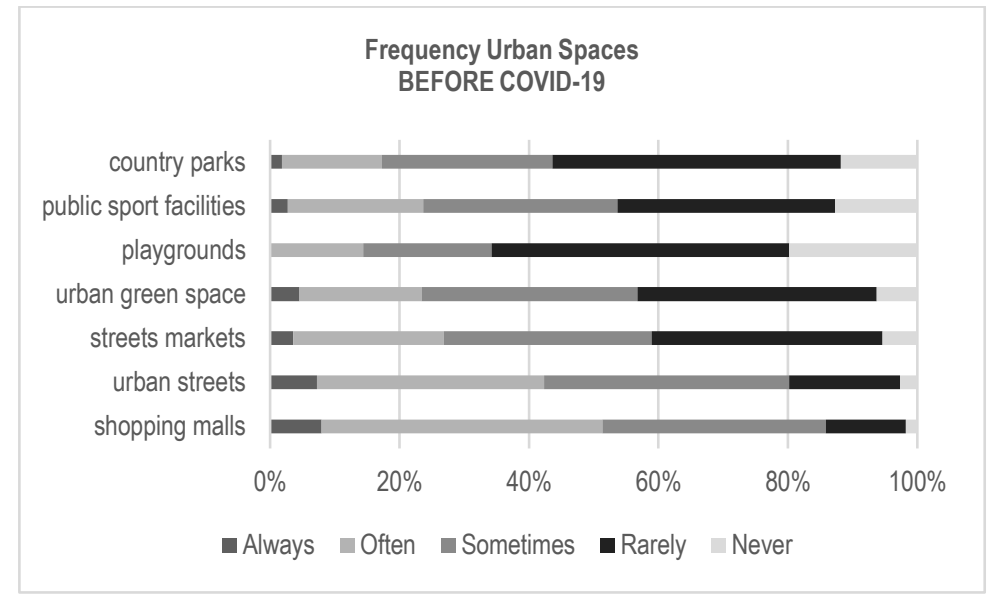

Fig. 4: Frequencies of urban spaces usage before the spread of Covid-19.

(source: Mirna Zordan)

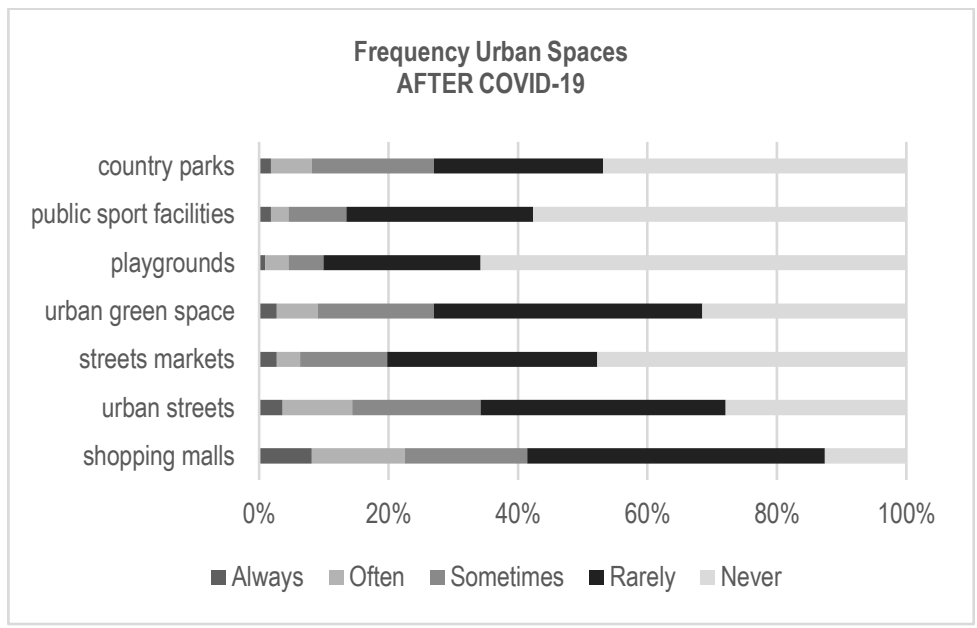

Fig. 5: Frequencies of urban spaces usage before the spread of Covid-19.

(source: Mirna Zordan)

\subsection{Preferences}

Survey respondents were asked to choose only one space among six typologies in which they were hanging out the most before the spread of Covid-19 and in which they are/would like to hang out now. Based on this question about decision making and preference of space, the null hypothesis $(\mathrm{HO})$ and the alternative hypothesis $(\mathrm{H} 1)$ previously set were as follow: $\mathrm{HO}=$ there is no preference in choosing some typologies of urban spaces among others; 
$\mathrm{H} 1=$ there is a preference in choosing some typologies of urban spaces among others;

Likert-type items 1 to 5 were tested performing Chi-square test. Results were positive and allowed the rejection of the null hypothesis at a 0.1 significance level $(p=70,8, d . f .=5)$. Survey respondents' preferences towards the use of urban spaces to hang out were mostly referred to shopping malls $(44 \%)$, followed by urban green spaces (19\%), public sports facilities (18\%), urban streets $(15 \%)$, street markets $(3 \%)$, and outdoor dining areas $(2 \%)$ before the spread of the Covid-19, see Fig. 4 . After the spread results significantly changed reverting the podium: urban green spaces (65\%), and malls $(17 \%)$. This result is revealing contrasting tendencies if compared to the actual usage of urban green spaces after the COVID-19 (see Fig. 3 and Fig.4), evidencing then the weakness and contrast between empirical evidence and actual preferences of urban dwellers. Other categories consistently dropped in preference, public sports facilities (9\%), urban streets $(7 \%)$, street markets (1\%), and outdoor dining areas reaching $0 \%$, see Fig.7. Of all participants, $63 \%$ ( $n=69)$, switched preference. Indepth interviews revealed that preferences related to shopping malls before the spread of the COVID-19 involved climate factors (summer too hot) and the presence of air-conditioning, convenience factors ("I can find everything I need there"), paired with the cleanliness of space. On the other hand, the switch to urban green spaces for the majority of respondents after the outbreak highlighted the need for lower levels of density, natural ventilation, the urge of exercise and physical activity, and self-healing processes related to the presence of green.

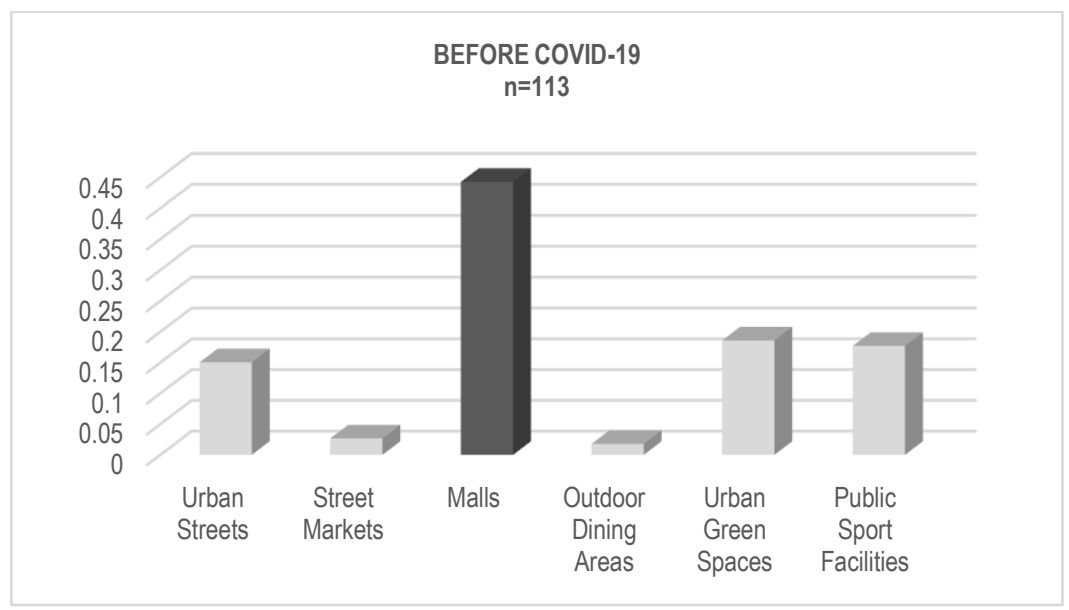

Fig. 6:Urban space preferences before the spread of Covid-19.

(source: Mirna Zordan) 


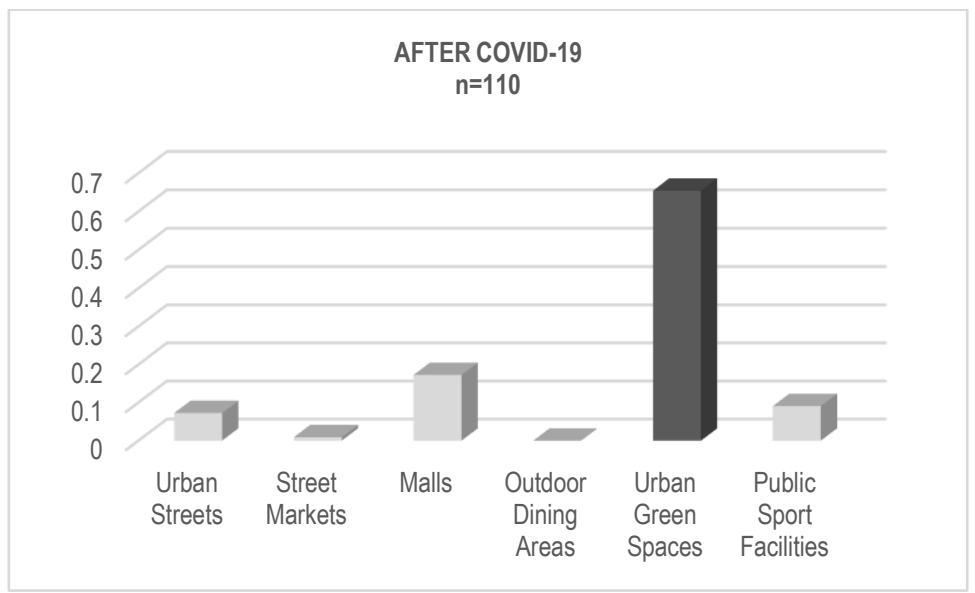

Fig. 7:Urban space preferences after the spread of Covid-19. (source: Mirna Zordan)

\subsection{Behavioural contagion}

to explore psychological dynamics affecting the decision-making process and to verify the eventual presence of behavioural contagion theory principles (assuming the high-degree liability of personal balance as already present due to the mass psychological distress condition), in-depth interviews were conducted among survey respondents' volunteers. All interviewees confirmed the presence of the first principle by stating that in a condition of imitative behaviour hesitation was prevalent in the decision (also supported by hesitation in replying to the question "If you go there because you are following them, how do you feel about going there?"). The third principle revealed to be the weakest as the strong urge toward the same behaviour of the initiator -in case of imitative behaviour-was not always present. In fact, participants tended to show willingness and urge towards activities but not towards the final destination: "I don't care too much if we are going there, I just follow them". Most of them followed initiators mostly for scope and not for place. In a few cases, even if finally going, they would rather go to another place: "I would rather go somewhere else". The fourth principles appeared to be the strongest as all initiators (before and after COVID-19) did not show any sign of fear or guilt in both answering the questions or actually deciding the destination. All the interviewees showed no hesitation and no sign of fear or guilt in replying questions related to self-initiative behaviour such as "If you decide to go, are you debated about going there?". Reversely, when imitative behaviour tends to appear, in the question "If you go there because you want to, are the others following you without hesitation?", hesitation resulted more evident, even if the final call consisted of going to the designated place.

The overall tendency showed that the majority of interviewees $(71 \%)$, was adopting selfinitiative behaviour (initiator) before Covid-19 and switched to imitative behaviour (imitator) after the spread. Simultaneously, participants who were following other people's choices before Covid-19 reverted their attitude by self-deciding their destination; however, those 
participants represented a minority $(29 \%)$. Most generally, none of the interviewees kept the same attitude before and after the Covid-19 outbreak and, as expected, the general tendency after the spread was to follow imitative behaviours dynamics and characteristics, with the consequence of a dramatic increase of levels of hesitation among the respondents.

\subsection{Discussion}

Following expectations, the overall trend of frequencies related to activities showed an increment of low and medium-low frequencies (rarely, never), and a dramatic decrease of high frequencies (always, often). Necessary activities drastically dropped but still survived, while social and optional activities experienced a more dramatic fall, bringing to a null value the possibly two most important activities for young generations: hanging out with friends and sports. Significantly, space preferences before the COVID-19 outbreak allowed the coexistence of social, optional and necessary activities, while the major shift in preferences of spaces after the spread reveals a more evident need of separation between the categories. Results of frequencies related to the effective usage of public spaces before and after the spread are actually in contrast with the questions related to preferences. Effective usages of spaces showed that urban green spaces decreased in frequency after the spread, while the question related to the possibility to choose and the actual preference of urban dwellers revealed a different outcome, namely the propensity to choose an urban green space rather than shopping malls or other typologies. This contrast between preferences and actual usages may question and address the possible weakness of empirical evidence or observation of reality without the support of perceptions related replies. The switch from malls towards urban green spaces indicates an expected preference of outdoor spaces, prioritising natural ventilation and natural sunlight, lower levels of density, and the need of self-healing processes related to the presence of green. This major overturn in priorities reveals the intrinsic importance of outdoor-related factors if compared to indoor-related ones: a result particularly significant in Hong Kong due to the weather conditions normally affecting urban dwellers choices. Normally, the prevalence of outdoor spaces preferences is not too high due to the adverse climate conditions (high temperature and levels of humidity). The separation of activities, and the preference, as expected, towards outdoor and environmental factors can have a strong repercussion in a high-density environment such as Hong Kong, where the scarcity of available land and its non-versatility represent an actual concern.

The testing of behavioural contagion principles' presence in the participants revealed positive results. Assuming the second principle (Wheeler, 1966) as already present due to mass distress conditions, the other principles seemed to be most likely satisfied after the spread except the third principle stating the strong urge towards individual satisfaction in line with the initiator. However, the prevalence of imitative behaviours after COVID-19 points out the weakening of self-initiative responses to urban preferences, highlighting the possible rising of optional and social activities related to groups. Besides the necessity of becoming versatile, implications towards the actual urban environment concern future needs of urban spaces capable of hosting more group-related activities. The underlining integration of mass 
psychological distress, and consequently of psychological factors affecting the urban population, into the design of future urban scenarios appear as inevitable.

\subsection{Limitations of the study}

Limitations related to the methodology concern sample size and targeted population for this pilot study. Data showed in this study represent initial portraits of tendencies, but do not allow accuracy of statements nor inferential analysis. Moreover, demographics data did not include typologies of households and living conditions, factors that may affect psychological and emotional states more than others. Another limitation of the study relies on the categorisation of urban spaces, as some categories were left out.Future directions should consider the implementation of the sample size and the widening of the population target to include other social groups; furthermore, the inclusion of questions related to living conditions and typologies of households should be added considering the sensitivity of the subject. Moreover, the research should become a longitudinal study to better capture variations and transition of human behaviour towards the use of urban space.

\subsection{Conclusion and Recommendations}

The impact of psychological mass distress on behavioural choices and preferences towards the use of urban spaces is a factor in need of more attention and exploration for future urban scenarios. Results of this study revealed that actual transitions in preference of urban spaces and activities, nonetheless in behavioural dynamics and related decision-making processes, significantly occur under a mass psychological distress condition. Results provided initial evidence about factors prioritised by young generations concerning the urban environment and dynamics of decision-making processes. More importantly, the contrast between questions related to actual uses of spaces and questions related to preferred choices addresses the need for deeper investigation in empirical evidence-related studies when human behaviour is involved. As appears in this pilot study, effective usage of public space does not represent urban dwellers needs nor preferences, especially regarding shopping malls and urban green spaces. The relevance of urban green spaces emerges here in line with the existing literature as a 'need for attention' factor influencing urban dwellers relief and wellbeing. Environmental factors in this study's results are prevailing above convenience and consumer-oriented solutions, suggesting a hypothetical questioning of the actual Hong Kong model. Moreover, this study advocates attention and more consideration of population needs in case of emergencies, aiming at redefining cities spaces capable of adaptation and survival. In fact, according to the preliminary results of this pilot study, solutions to provide more versatile and flexible spaces have to be explored under the light of initial tendencies revealed in this study. Future challenges and research should include more accurate investigation of psychological needs and perceptions, and in-depth exploration and inclusion of mass psychological distress into future urban design guidelines and policies to achieve efficient and resilient criteria for better urban scenarios. 


\section{Acknowledgements}

We would like to thank all volunteers and survey participants, nonetheless all colleagues and people that helped with the spread of the survey.

\section{References}

Allen, J., Muñoz, J. C., \& Ortúzar, J. de D. (2019). On evasion behaviour in public transport: Dissatisfaction or contagion? Transportation Research Part A: Policy and Practice, 130(August), 626-651. https://doi.org/10.1016/j.tra.2019.10.005

Amin, A. (2008). Collective culture and urban public space. City, 12(1), 5-24. https://doi.org/10.1080/13604810801933495

Bish, A., \& Michie, S. (2010). Demographic and attitudinal determinants of protective behaviours during a pandemic: A review. British Journal of Health Psychology, 15(4), 797-824. https://doi.org/10.1348/135910710X485826

Bishop, K., \& Marshall, N. (2017). Social Interactions and the Quality of Urban Public Space. In Encyclopedia of Sustainable Technologies (Vol. 2, Issue 2007). Elsevier. https://doi.org/10.1016/B978-0-12-409548-9.10177-0

Boone, H. N. J., \& Boone, D. A. (2012). Analyzing Likert Data. Journal of Extension, 50(2), 207-209. https://doi.org/10.1016/j.jfma.2016.04.007

Carmona, M. (2010). Contemporary public space, part two: Classification. Journal of Urban Design, 15(2), 157-173. https://doi.org/10.1080/13574801003638111

Carnap, R. (1936). Testability and Meaning. Philosophy of Science, 3(4), 419-471.

Carnap, R. (1937). Testability and Meaning_Continued. Philosophy of Science, 4(1), 1-40.

Dodds, P. S., \& Watts, D. J. (2004). Universal behavior in a generalized model of contagion. Physical Review Letters, 92(21), 1-4. https://doi.org/10.1103/PhysRevLett.92.218701

Epstein, J. M., Parker, J., Cummings, D., \& Hammond, R. A. (2008). Coupled contagion dynamics of fear and disease: Mathematical and computational explorations. PLOS ONE, 3(12). https://doi.org/10.1371/journal.pone.0003955

Francis, J., Giles-Corti, B., Wood, L., \& Knuiman, M. (2012). Creating sense of community: The role of public space. Journal of Environmental Psychology, 32(4), 401-409. https://doi.org/10.1016/j.jenvp.2012.07.002

Freedman, J. L., Birsky, J., \& Cavoukian, A. (1980). Environmental Determinants of Behavioral Contagion: Density and Number. Basic and Applied Social Psychology, 1(2), 155-161. https://doi.org/10.1207/s15324834basp0102_4

Gehl, J. (1987). Life Between Buildings (6th ed.). The Danish Architectural Press. https://books.google.com.tr/books? id=K98JAQAAMAAJ\&q=9788774073604\&dq=9788774073604\&hl=en\&sa=X\&v ed=0ahUKEwiTIJvbi6vcAhUFkiwKHWwmBm0Q6AEIKTAA

Ghel, J., \& Svarre, B. (2013). How to Study Public Life. Island Press. 
Hanan, H. (2017). Every Day Practices and Experiential Urban Space. Asian Journal of Environment-Behaviour Studies, 2(5), 27. https://doi.org/10.21834/aje-bs.v2i5.220

Hatfield, E., Cacioppo, J. T., \& Rapson, R. L. (1992). Primitive emotional contagion. Emotion and Social Behavior, August, 151-177.

Huremovic, D. (2019). Psychiatry of Pandemics. A Mental Health Response to Infection Outbreak. Springer.

Jones, J. H., \& Salathé, M. (2009). Early assessment of anxiety and behavioral response to novel swine-origin influenza a(H1N1). PLoS ONE, 4(12), 2-9. https://doi.org/10.1371/journal.pone.0008032

Kohn, M. (2004). Brave new neighborhoods. The privatization of public space. Routledge. https://doi.org/10.4324/9780203495117

Le Bon, G. (1896). The Crowd. A study of the popular mind. In 2001. Batoche Books.

Mehta, V. (2006). Lively Streets: EXPLORING THE RELATIONSHIP BETWEEN BUILT ENVIRONMENT AND social behavior. University of Maryland.

Mehta, V. (2008). Walkable streets: Pedestrian behavior, perceptions and attitudes. Journal of Urbanism, 1(3), 217245. https://doi.org/10.1080/17549170802529480

Mehta, V., \& Bosson, J. K. (2018). Revisiting Lively Streets: Social Interactions in Public Space. Journal of Planning Education and Research. https://doi.org/10.1177/0739456X18781453

Nasution, A. D., \& Zahrah, W. (2018). Quality of Life : Public open space effects. Asian Journal of EnvironmentBehaviour Studies, 3(10), 124. https://doi.org/10.21834/aje-bs.v3i10.319

Polansky, N., Lippitt, R., \& Redl, F. (1950). An investigation of behavioral contagion in groups. Human Relations.

Redl, F. (1949). The phenomenon of contagion and "shock effect" in group therapy. In Searchlights on delinquency; new psychoanalytic studies. (pp. 315-328). International Universities Press.

Rubin, G. J., Amlôt, R., Page, L., \& Wessely, S. (2009). Public perceptions, anxiety, and behaviour change in relation to the swine flu outbreak: Cross sectional telephone survey. BMJ (Online), 339(7713), 156. https://doi.org/10.1136/bmj.b2651

Saunders, M., Lewis, P., \& Thornhill, A. (2009). Research Methods for business students (5th ed.). Pearson Education Limited.

Shirado, H., Crawford, F. W., \& Christakis, N. A. (2020). Collective communication and behaviour in response to uncertain 'Danger' in network experiments. Proceedings of the Royal Society A: Mathematical, Physical and Engineering Sciences, 476(2237). https://doi.org/10.1098/rspa.2019.0685

Suzuki, S., Jensen, E. L. S., Bossaerts, P., \& O’Doherty, J. P. (2016). Behavioral contagion during learning about another agent's risk-preferences acts on the neural representation of decision-risk. Proceedings of the National Academy of Sciences of the United States of America, 113(14), 3755-3760. https://doi.org/10.1073/pnas.1600092113

UN-Habitat. (2013). Streets as Public Spaces and Drivers of Urban Prosperity.

van Baaren, R. (2007). Encyclopedia of Social Psychology. In Encyclopedia of Social Psychology. SAGE Publications. https://doi.org/10.4135/9781412956253 
Villanueva, K., Badland, H., Hooper, P., Koohsari, M. J., Mavoa, S., Davern, M., Roberts, R., Goldfeld, S., \& GilesCorti, B. (2015). Developing indicators of public open space to promote health and wellbeing in communities. Applied Geography, 57, 112-119. https://doi.org/10.1016/j.apgeog.2014.12.003

Wheeler, L. (1966). Toward a theory of behavioral contagion. Psychological Review, 73(2), 179-192. https://doi.org/10.1037/h0023023

White, S. C. (2010). Analysing wellbeing: A framework for development practice. Development in Practice, 20(2), 158-172. https://doi.org/10.1080/09614520903564199

White, W. H. (1980). The Social Life of Small Urban Spaces (P. for P. S. Inc (ed.)). Project for Public Spaces Inc.

Zordan, M. (2018). Ground China. Starting from Villages. In Walkable Cities in High-Density China. Livable, Healthy and Sustainable (pp. 41-81). Tongji University Press. 